\title{
Orthopedic Approach to the Early Management of Pelvic Injuries
}

\author{
Michael J. Weaver • Marilyn Heng
}

Published online: 25 January 2015

(C) Springer International Publishing AG 2015

\begin{abstract}
High-energy pelvic fractures in trauma patients cause significant morbidity and mortality. A systematic approach to the early evaluation and management of these patients leads to improved outcomes. The initial care of patients with a displaced pelvic fracture and hemodynamic instability should focus upon three areas: 1) resuscitation, 2) bony stability, and 3) hemorrhage control. Aggressive resuscitation with blood products and clotting factors is paramount. The initial reduction of the pelvic ring and temporary stabilization can be achieved through the use of pelvic sheeting or binder placed in the field or trauma bay. Urgent surgical stabilization with external or internal fixation is often required. Hemorrhage control can be achieved through pelvic packing and/or angioembolization. The initial management of displaced pelvic fractures is multidisciplinary and should involve close collaboration between trauma surgery, orthopedic surgery, anesthesia, and interventional radiology.
\end{abstract}

Keywords Pelvic fracture $\cdot$ Polytrauma $\cdot$ Resuscitation . Hemorrhage $\cdot$ Pelvic packing $\cdot$ Retroperitoneal packing . Angiography $\cdot$ Pelvic binder

\section{Introduction}

High-energy displaced pelvic fractures represent a significant cause of mortality and morbidity in the trauma patient. Mortality in trauma patients with a pelvic fracture has been reported to range from 5 to $20 \%$ in all-comers [1,2], with higher

This article is part of the Topical Collection on Trauma to the Pelvis

M. J. Weaver $(\bowtie) \cdot$ M. Heng

Department of Orthopaedic Surgery, 75 Francis Street, Boston, MA 02115, USA

e-mail:MWEAVER@research.bwh.harvard.edu rates in patients with concomitant hemodynamic instability or open injury [2-4].

An understanding of the mechanisms of injury that lead to pelvic fractures is useful to guide treatment and understand patterns of injury and causes of mortality. While fractures that result from a head-on impact are associated with significant blood loss, side-pact injuries are often seen in conjunction with head and abdominal injuries $[5,6]$.

The initial management of displaced pelvic fractures is multimodal and should involve close collaboration between trauma surgery, orthopedic surgery, anesthesia, and interventional radiology $[7,8]$. The immediate care of patients with unstable pelvic fracture should be focused on three primary areas: resuscitation, bony stability, and hemorrhage control.

\section{Evaluation}

Patients with a suspected pelvic fracture should be managed according to the principles of Advanced Trauma Life Support (ATLS) [9]. Of primary concern is establishing and maintaining a viable airway while maintaining $\mathrm{C}$-spine control, evaluation of the patient's breathing with maintenance of adequate oxygenation, and protection of the patient's circulation with assessment for hemorrhage and adequate control of bleeding.

During the primary survey, the stability of the pelvis should be assessed [9]. Although physical examination alone without fluoroscopy is not sensitive to detect minor instability, the presence of gross instability portends a severely unstable fracture that may be associated with significant bleeding. The stability of the pelvis is assessed by firmly grasping the iliac wings and assessing for any motion by attempting to push and pull them apart and together [10॰]. A significant leg length discrepancy in the absence of an obvious long bone fracture may indicate a vertically unstable and displaced pelvic fracture. 
During the secondary survey, the examiner should fully examine the pelvis. Care should be taken to look for open wounds around the pelvis and perineum that may indicate an open injury (Fig. 1). All patients should have a digital rectal examination, and female patients should have a vaginal examination to assess for lacerations that could communicate with a pelvic fracture. Open pelvic injuries are associated with mortality of up to $50 \%[11 \cdot 12,13]$. Antibiotics including a firstgeneration cephalosporin and a broad-spectrum antibiotic with gram-negative coverage such as gentamycin should be given promptly to reduce the risk of infection [14]. Large perineal or rectal injuries may necessitate a diverting colostomy to avoid contamination of the wounds $[15,16]$.

The presence of blood at the urethral meatus may suggest a urethral injury, and in these cases, a retrograde urethrogram (RUG) should be obtained prior to Foley catheterization [17]. Once passed, the presence of blood in the urine may indicate a bladder injury, and a cystogram should be performed $[18,19]$.

Obtaining an AP radiograph of the pelvis in the trauma bay is essential in the treatment of a hemodynamically unstable patient with a suspected pelvic fracture. This radiograph alone is enough to direct the initial management of displaced pelvic ring injuries. Some authors have advocated deferring an AP pelvis radiograph if the suspicion of a pelvic fracture is low and the patient is stable enough for computed tomography of the abdomen and pelvis [20-22]. However, in cases where a pelvic ring injury is likely or the patient is hemodynamically unstable, an AP plain radiograph should be taken as part of the initial primary survey evaluation to allow for the initiation of treatment as soon as possible.

The initial management of a displaced pelvic fracture requires only an AP radiograph; however, definitive treatment most often requires a CT scan to better delineate the posterior injury.

Consultation with the orthopedic trauma service should be initiated as soon as a pelvic fracture is suspected. The successful evaluation and management of these injuries requires a multidisciplinary approach $[7,8]$.

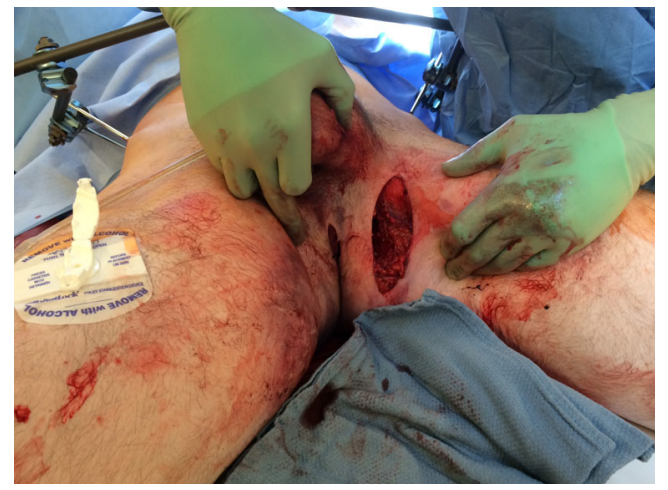

Fig. 1 A patient with a large perineal laceration that communicates with the rectum. A diverting colostomy was required to prevent stool soiling of the wound

\section{Classification}

While there are numerous classification systems used to describe pelvic fractures, the most common is the Young and Burgess system [23]. This classification system is based upon the mechanism of injury that leads to predictable patterns of injury and displacement $[6,23]$. It is useful in describing injuries and also to help guide both initial treatment and definitive fixation (Fig. 2).

Anteroposterior Compression (APC). This pattern of injury results from a direct blow to the anterior pelvic ring or occasionally from an external rotation force applied to one lower limb. The common term for this type of injury is "open book" as the anterior pelvic ring separates and opens, while the posterior ligamentous complex of the sacroiliac (SI) joints remains intact, allowing the pelvis to hinge open. APC 1 injuries involve only minor $(<2.5 \mathrm{~cm})$ pubic diastasis. APC 2 injuries involve more pubic diastasis $(>2.5 \mathrm{~cm})$ but have intact posterior SI ligaments. APC 3 injuries involve significant pubic diastasis and the complete disruption of the posterior SI ligaments. APC injuries permit the expansion of the pelvic ring resulting in a significant increase in pelvic volume. They are often associated with significant hemorrhage, which is the most common cause of death in patients with displaced APC injuries [6].

Lateral Compression (LC). This is the most common injury pattern. It often results from a side-impact motor vehicle collision. A laterally directed force causes the internal rotation of the injured hemipelvis with a reduction in pelvic volume. LC 1 injuries involve superior and inferior pubic ramus fractures with

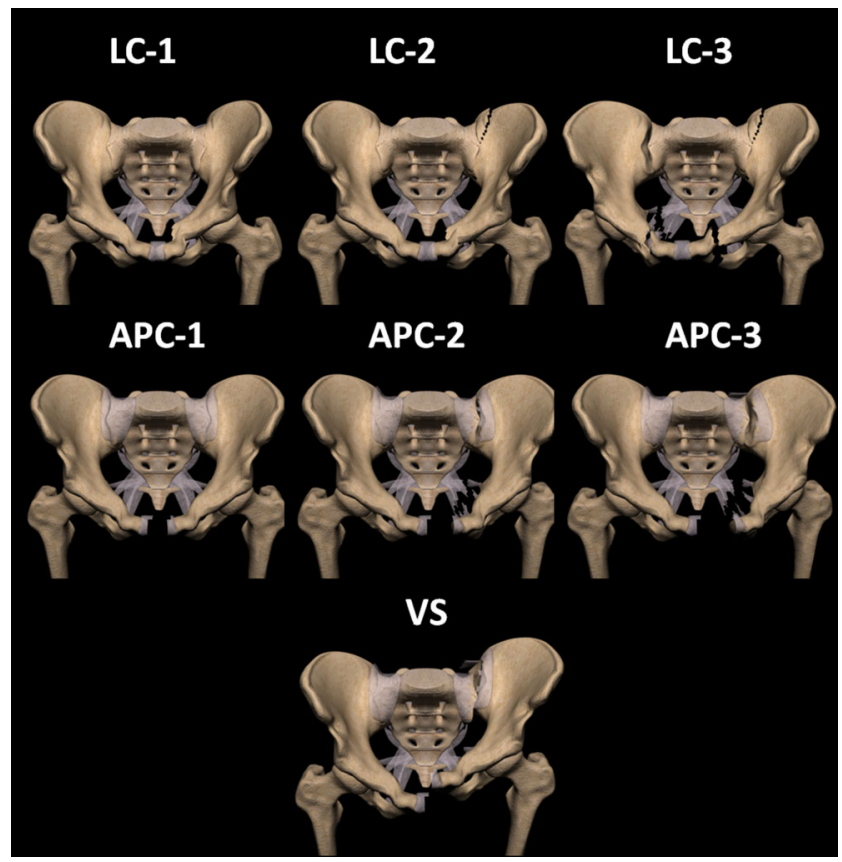

Fig. 2 The Young and Burgess Classification of pelvic fractures 
a sacral ala fracture that is often incomplete. LC 2 injuries involve superior and inferior pubic ramus fractures and a fracture/dislocation of the SI joint on the involved side. LC 3 injuries involve a lateral compression-type injury on one side, with an APC-type injury on the contralateral side, and are commonly called "windswept" pelvic fractures. Significant hemorrhage from these injuries is possible, although less common, and is typically the result of arterial injury. The most common causes of mortality in patients with LC-type fracture patterns are associated injuries such as head and abdominal injuries [6].

Vertical Shear (VS). These injury patterns result from falls from a significant height but also occasionally from motor vehicle collisions [6]. The injury can be either ligamentous or bony or a combination of both. In either case, the hallmark of this injury is the proximal migration of one hemipelvis. Vertical shear fractures are typically very unstable. They often require the placement of skeletal traction to partially reduce the displaced hemipelvis prior to definitive management.

Combined Mechanism. These rare injuries are patterns that do not fit into the other categories. Associated acetabular fractures are often present. These injuries are best described anatomically, and treatment is tailored to the specific pattern of injury and displacement.

\section{Management}

The management of displaced pelvic fractures should be multimodal and protocol driven. It has been shown that the use of aggressive management protocols can reduce transfusion rates and mortality [24, 25••, 26, 27].

The initial management of displaced pelvic fractures in hemodynamically unstable patients involves three domains (Fig. 3):

\section{Resuscitation \\ 2. Bony stability \\ 3. Hemorrhage control}

These three domains do not need to be addressed in sequence but, in practice, can be addressed in parallel.

\section{Resuscitation}

The pelvic cavity and retroperitoneal space represent a large potential space for exsanguination in the trauma patient. Cadaveric study has shown that the potential volume of the retroperitoneum with an intact pelvis is approximately $5 \mathrm{~L}$,

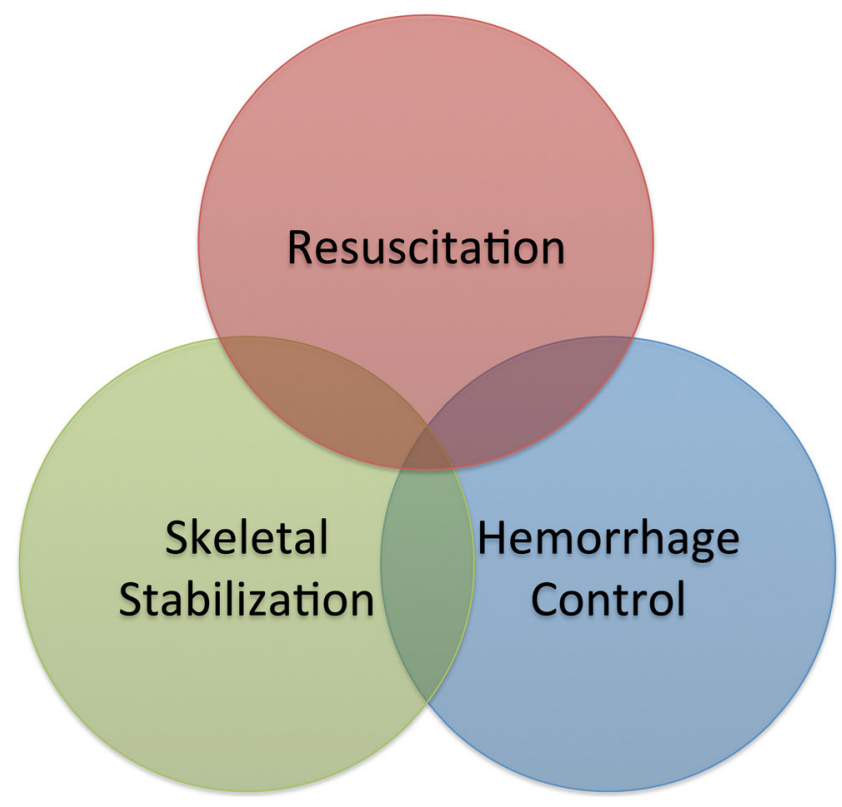

Fig. 3 Three areas of focus during the acute management of pelvic fractures

and with the disruption of the bony anatomy, such as with an open-book pelvic fracture, several times, the total body blood volume can be lost into this expanding space [28]. The proper resuscitation of the trauma patient with a pelvic injury is vital to their survival.

Resuscitation begins in the prehospital phase, and usually, by the time a hemodynamically unstable pelvic fracture patient arrives in the trauma bay, they have had an adequate trial of fluid resuscitation with $1 \mathrm{~L}$ crystalloid fluid as per the most recent edition of the Advanced Trauma Life Support (ATLS) protocol [9]. The goal of resuscitation is to prevent the development of the lethal combination of acidosis, hypothermia, and coagulopathy. The prevention of hypothermia is an important aspect of resuscitation. Judicious exposure and remembering to re-cover the patient after the examination of the pelvis is key but often overlooked.

Permissive hypotension is a term used to denote the acceptance/target of a lower blood pressure in trauma patients until hemorrhage control is achieved. Its premise is in avoiding the development of a dilutional coagulopathy in the patient. There have been no experimental or observational studies focused on permissive hypotension in pelvic fracture patients specifically. A 2011 randomized controlled trial of trauma patients with hemorrhagic shock assigned patients requiring emergent surgery to a low mean arterial pressure (target MAP $50 \mathrm{mmHg}$ ) treatment arm vs a control arm of standard resuscitation to a target MAP of $65 \mathrm{mmHg}$ [29••]. Their preliminary results show significantly lower mortality in the early postoperative period for patients managed with a hypotensive goal. The risk of coagulopathy in the low mean arterial pressure group was lower as was the severity of coagulopathy if it did occur [29••]. There is concern regarding the use of 
permissive hypotension in certain populations, specifically patients with head injury and elderly patients who have a higher likelihood for coronary artery disease - both of these types of patients can commonly present with an associated pelvic fracture. A recent retrospective study of the TraumaRegister DGU in Germany looked specifically at permissive hypotension in elderly trauma patients [30]. There was no difference in mortality seen between elderly patients treated with preclinical fluid resuscitation of $\leq 1 \mathrm{~L}$ fluid and those treated with $>1 \mathrm{~L}$ fluid resuscitation. It also appeared that patients in the low volume resuscitation group had a shorter prothrombin ratio (analogous to INR) indicating less coagulopathy. Thus, restraint in fluid resuscitation even in elderly patients may be safe.

Current literature emphasizes the importance of the early use of blood and blood products in resuscitation efforts. The concepts of "damage control resuscitation" first developed through the military experience in Iraq and Afghanistan have led to the general acceptance of a 1:1:1 plasma:platelets:RBCs transfusion protocols [31, 32]. These protocols have been traditionally utilized in the context of the massively transfused patients ( $\mathrm{RBC}$ requirement of $\geq 10$ units in $24 \mathrm{~h}$ ); however, more recent studies have begun to apply these principles to all trauma patients requiring transfusion. In a 2013 study of 905 trauma patients, the Prospective, Observational, Multicenter, Major Trauma Transfusion (PROMMTT) investigators demonstrated an association of decreased mortality within the first $24 \mathrm{~h}$ with higher plasma:RBC transfusion ratios and within the first $6 \mathrm{~h}$ with higher platelet:RBC transfusion ratios [33].

Age, systolic blood pressure, shock on arrival, revised trauma score (RTS), and base deficit have been identified through retrospective analysis as predictors for transfusion in pelvic fracture patients [2]. The influence of fracture pattern on transfusion requirement has been examined. Traditionally, APC 3 fractures as classified by the Young-Burgess Classification have been shown to require the greatest amounts of fluid resuscitation in the first $24 \mathrm{~h}$ [23]. In the modern era of resuscitation, both the Young-Burgess Classification and the Tile Classification for pelvic fractures have been shown to be predictive of transfusion requirements in general $[34,35]$; however, fracture pattern alone cannot solely indicate which patients require transfusion or not as only $40-60 \%$ of pelvic fractures with bony instability require transfusion, and conversely, some pelvic fractures deemed skeletally stable will require transfusion [36].

\section{Bony Stability}

Urgent reduction and temporary stabilization of displaced pelvic fractures is paramount. Displaced pelvic fractures are associated with the disruption of the rich venous plexus in the presacral area and pelvic floor [37]. Fractured bone surfaces are also a significant source of blood loss. Injury patterns can increase the pelvic volume such that the hemorrhage of the multiples of the circulating blood volume into the true pelvis and retroperitoneal space can occur [28]. Furthermore, displaced pelvic fractures are most often unstable, and the movement of fracture fragments can disrupt clots that form as a response to abate hemorrhage. The reduction of displaced pelvic fractures reduces the pelvic volume, re-opposes fractured bone surfaces, and allows for the formation of clot to reduce bleeding.

The easiest and least invasive way to reduce the pelvic volume and temporarily stabilize the pelvis is with circumferential sheeting or the application of a pelvic binder [38• 39]. Emergency personnel can perform this procedure in the field, or it can be performed upon initial presentation in the trauma bay if a displaced pelvic fracture is suspected [38•]. It is our recommendation that a sheet/binder be applied in all cases of suspected pelvis fracture and then its need be re-assessed after the primary survey and the evaluation of the pelvic radiograph and fracture pattern.

Care should be taken to ensure that the sheet or binder is centered squarely over the greater trochanters and securely tightened (Fig. 4). Postreduction radiographs are important to gauge the reduction and effectiveness of the sheet or binder. There is some controversy as to the efficacy of using sheet or binder stabilization in the setting of lateral compression injuries; however, the risk is likely very low, and unless the fracture pattern is known, sheeting or a binder should be employed.

If a sheet or binder is applied to a patient with a suspected pelvic fracture, but radiographs reveal no injury, an additional set of films should be taken immediately following the removal of the binder or sheet to ensure that a purely ligamentous injury was not reduced by the sheet and missed on the initial radiographs. To avoid the complication of pressure ulcers and skin breakdown, a binder or sheet should not be left on for more than $24 \mathrm{~h}$.

External fixation is the most commonly employed technique for achieving temporary reduction and stabilization of displaced pelvic ring injuries. The technique involves the placement of Schanz pins into the innominate bones of the

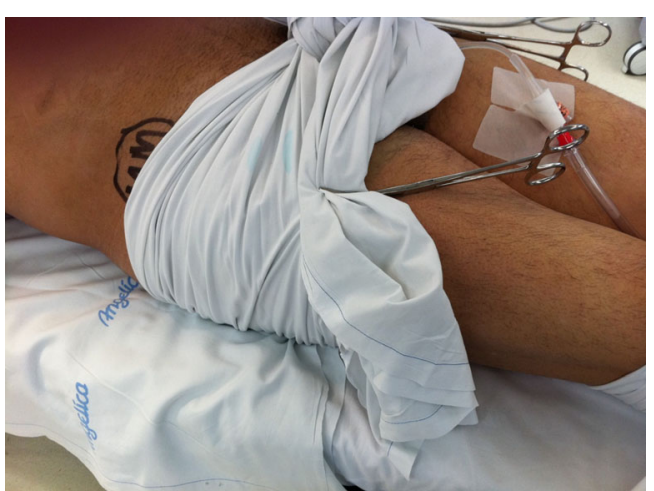

Fig. 4 A circumferential pelvic sheet or binder should be centered on the greater trochanters and cinched tight. Additionally, the toes should be taped together to help avoid the external rotation of the injured limb and hemipelvis 
pelvis [40]. The pins are then used to directly manipulate the fractured fragments, and an external frame is then constructed to maintain this reduction. Pins can be safely placed into the iliac crest without the aide of C-arm fluoroscopy (Fig. 5). Supra-acetabular pin placement is also useful, although this technique requires the use of $\mathrm{C}$-arm fluoroscopy to ensure safe pin placement.

External fixation is typically used to stabilize the bony pelvis and affects an approximate reduction of the bone fragments [40]. In many cases, once the patient has been stabilized, the frame can be removed and the pelvis can be repaired through an open approach [41]. This allows for a direct reduction of the fracture fragments and avoids the problems associated with long-term external fixation such as pin site infection and a bothersome contraption on the patient [42]. Occasionally, the external fixator is used for definitive fixation, and in these cases, it is often left in place for 8-12 weeks.
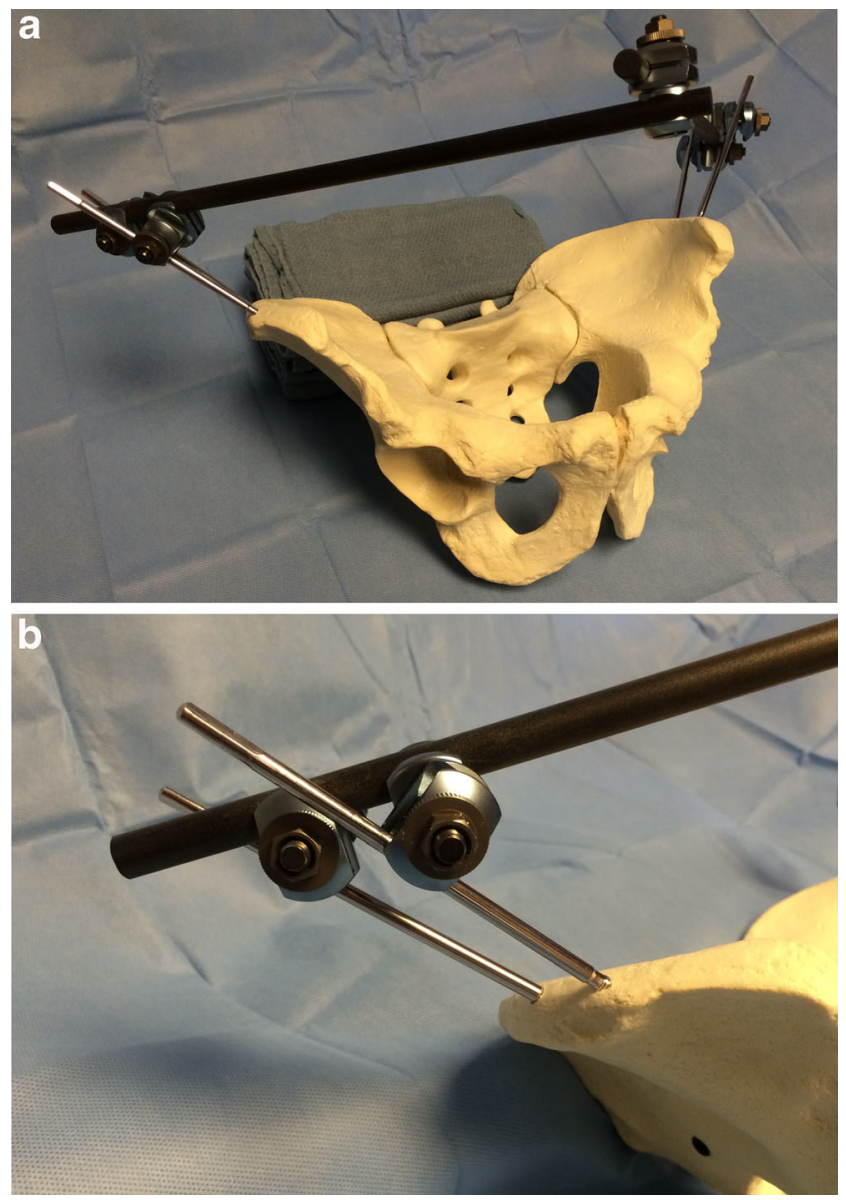

Fig. 5 External fixation involves the placement of pins into the innominate bones (a). In this case, the pins are placed into the iliac crest (b). An anterior frame is then constructed to maintain the reduction of the pelvis. This frame can be adjusted to allow for access to the abdomen if required. External fixation provides the excellent control of anterior displacement but does not allow for reduction or stabilization of injuries that involve significant posterior displacement
While the external fixation of the iliac wings is excellent at controlling the anterior pelvic ring and managing fracture patterns that involve primarily anterior displacement or "open book"-type injuries, injuries that involve the significant displacement of the posterior pelvic ring are often poorly controlled with external fixation alone [43, 44]. External fixator pins are placed into the anterior ring, and given the long lever arm between the posterior ring and the anterior frame, there is little ability to directly control the posterior pelvic ring. In these situations, the use of a "C-clamp" is advantageous. This device involves the placement of two pins, one through the outer table of each ilium, in the area of the sacroiliac joint. An external frame then allows direct compression across the posterior aspect of the pelvis (Fig. 6) [45, 46].

Some authors advocate for urgent or emergent open reduction and internal fixation of displaced pelvic ring injuries [47]. This is particularly useful in the setting of anteroposterior compression (APC) injuries. The surgical approach is simple, and the reduction and fixation of these injuries can often be
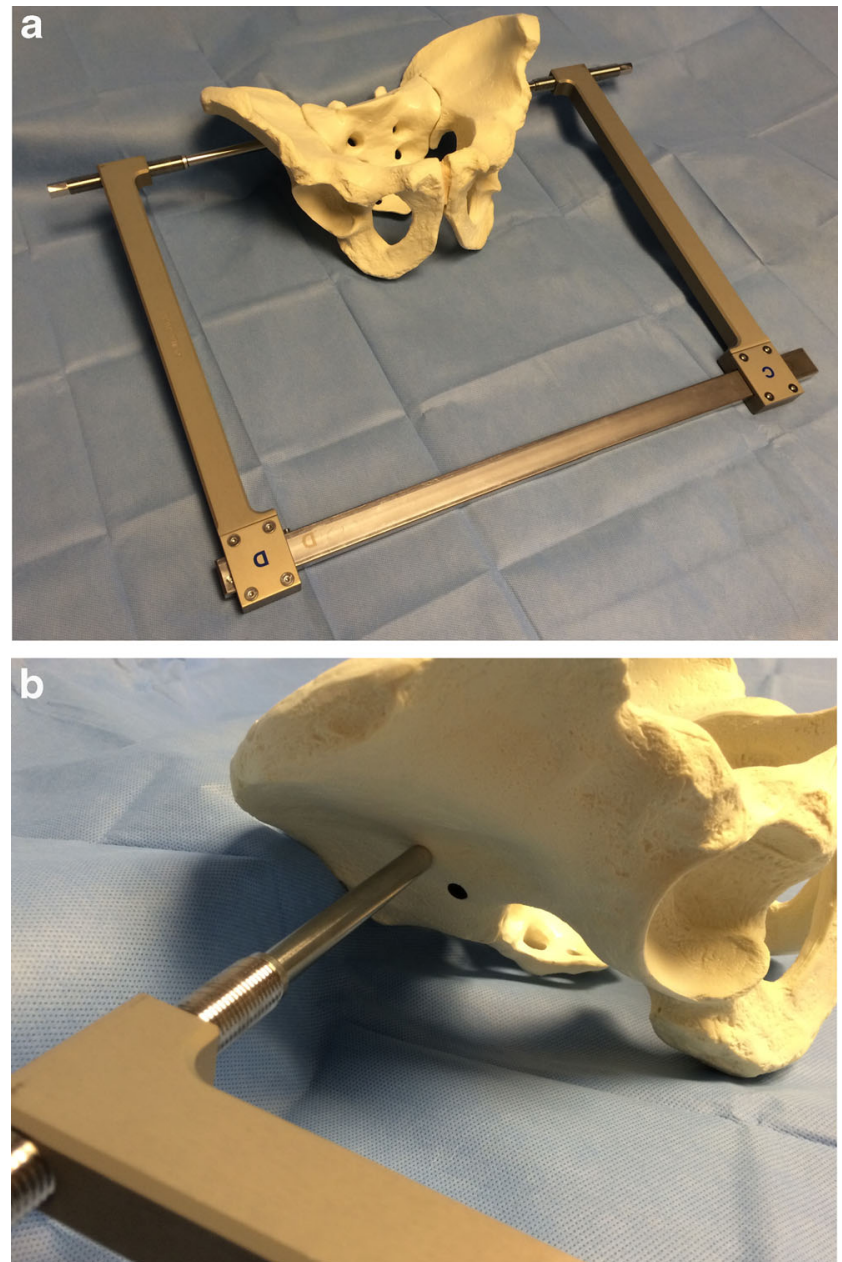

Fig. 6 The C-clamp is useful in controlling fractures with significant posterior displacement (a). In this case, specialized pins (b) are placed at the level of the sacroiliac (SI) joint, and direct manipulation and compression of the posterior pelvis is possible 
accomplished as fast as external fixation. Often, this treatment strategy is combined with retroperitoneal packing (Fig. 7). Depending on the quality of the reduction and the method of internal fixation, this technique can be used as the definitive mode of fixation or can be revised if required during subsequent visits to the operating room.

\section{Hemorrhage Control}

One of the more recent controversies in the early orthopedic management of pelvic fractures in the trauma patient has been the algorithm for management of the "exsanguinating," i.e., hemodynamically unstable pelvic fracture patient.
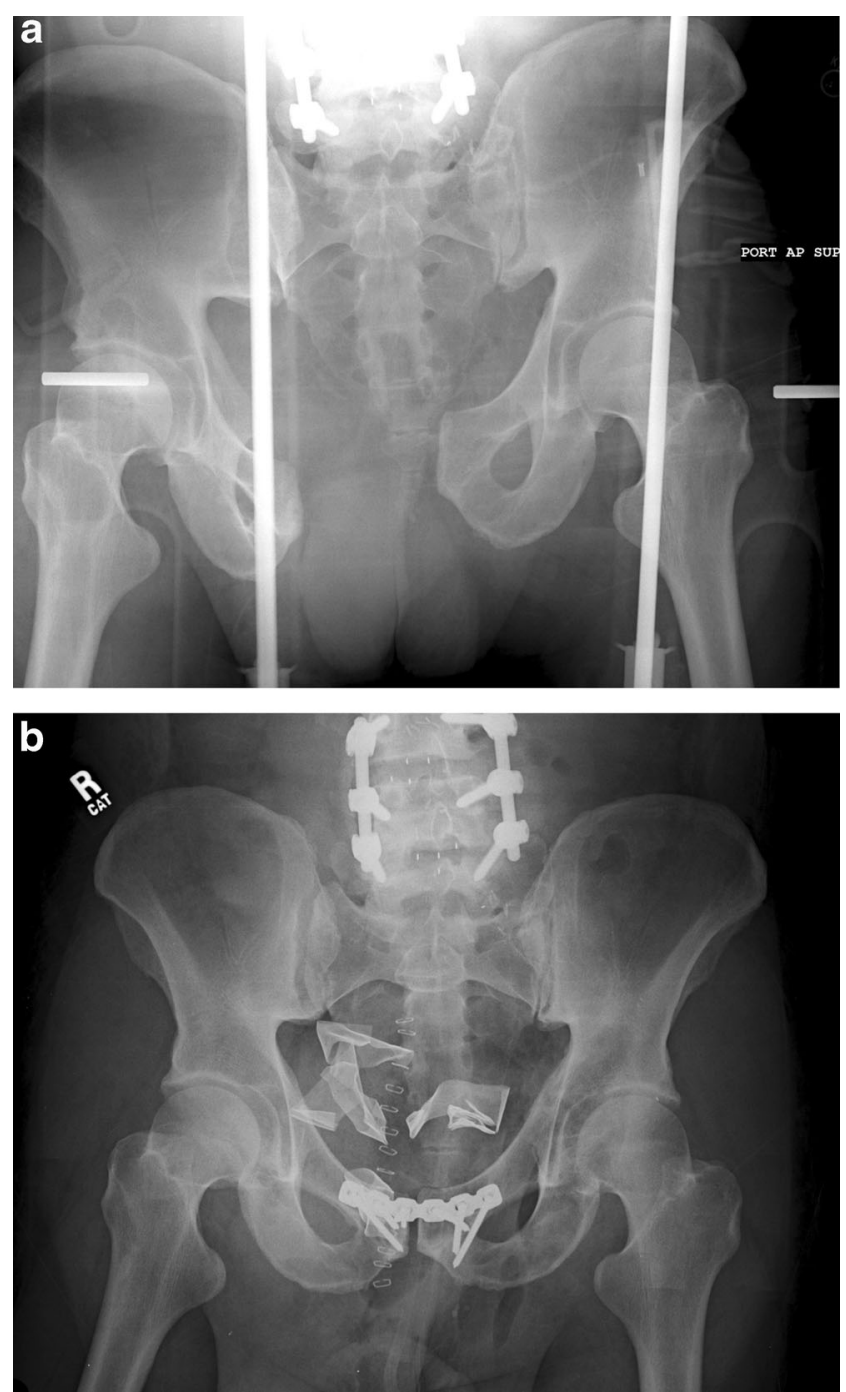

Fig. 7 Preoperative (a) and postoperative (b) radiographs of a patient with an APC 3 injury. Note the initial pubic symphysis diastasis and rightsided SI joint widening. The patient was initially treated with open reduction and internal fixation with retroperitoneal packing. The anterior pelvic ring has been reduced, but there is still the subtle widening of the SI joint that will require definitive treatment with a SI screw. Multiple packs are seen within the true pelvis
Traditionally, advocacy for the use of angioembolization has been strong in North America, whereas the use of surgical pelvic packing is more popular in Europe.

Surgical pelvic packing consists of preperitoneal pelvic packing. This may be done through a limited Pfannenstiel incision, and packing within the retroperitoneum is performed without the violation of the peritoneum. Care must be taken to ensure the packs are placed inferior, down into the true pelvis, such that they can tamponade the presacral area and pelvic floor. It is important that the bony stabilization of the pelvis is obtained before preperitoneal packing to allow the packing to achieve a tamponade against the pelvic rim. This technique is explicitly different from the older methods of surgical pelvic packing which frequently occurred transperitoneal through a laparotomy incision and which had suboptimal results [48, 49].

Within North America, there has been recent increased interest in preperitoneal packing for hemodynamically unstable pelvic fracture patients before angioembolization. One case series at a center that has incorporated preperitoneal packing into their treatment algorithm since 2004 has reported their results noting that preperitoneal packing has been effective in the control of hemorrhage in hemodynamically unstable pelvic fracture patients [50]. During their 5.5-year study period, no pelvic fracture patients died from acute hemorrhage. The

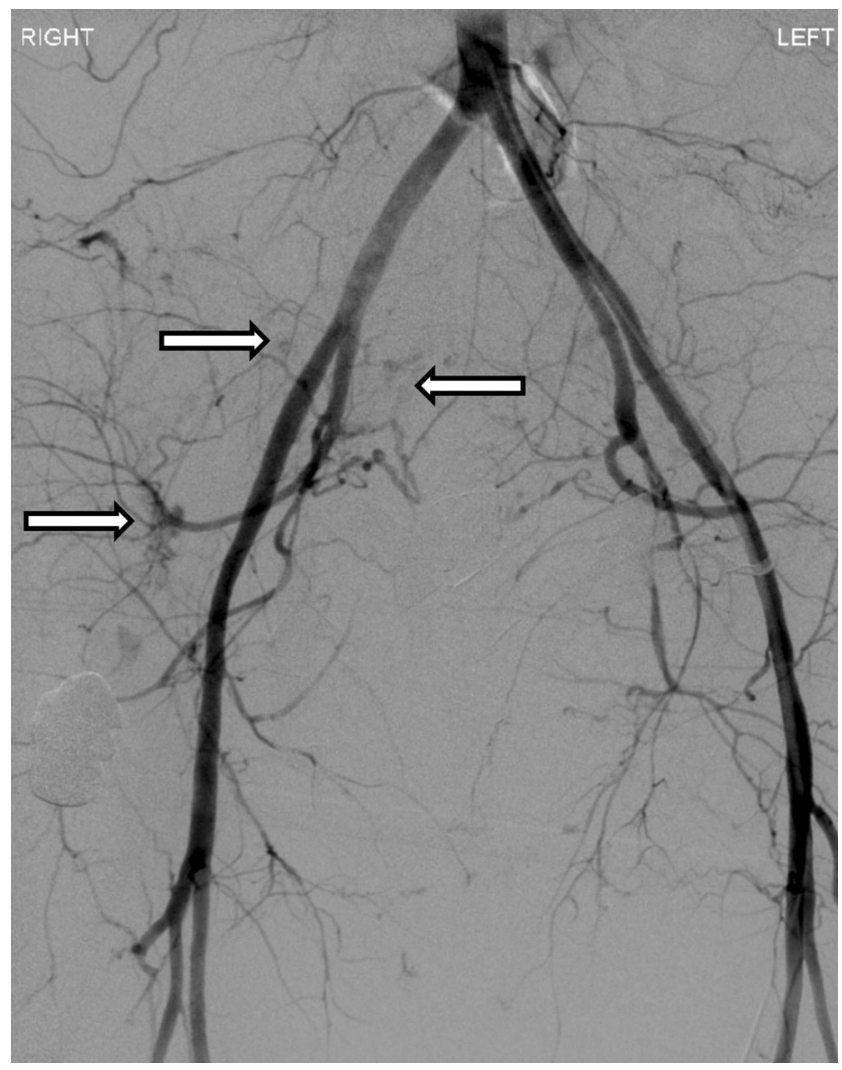

Fig. 8 The angiography of a patient with an unstable pelvic fracture. Note the extravasation of contrast around the branches of the right internal iliac artery and superior gluteal artery (arrows) 
use of angioembolization secondarily was required in $13 \%$ of those patients who underwent pelvic packing.

Indications for angiographic embolization have included contrast extravasation on arterial phase CT scans and ongoing hemodynamic instability despite fluid and blood resuscitation (Fig. 8). Fracture pattern alone does not necessarily aide in determining the utility of angiography [51]. The efficacy rates of arterial embolization for pelvic fracture have been reported from 81 to $100 \%$ with the need for repeat embolization in approximately $10 \%$ of cases [52].

There have been no randomized controlled trials evaluating angiographic embolization vs preperitoneal pelvic packing in the literature. One observational before-and-after cohort study examined the outcomes after a treatment algorithm shift from emergency angiographic embolization to preperitoneal packing [53・•]. This study demonstrated shorter median time to operating room compared to mean time to angiography suite. There was a significant decrease in blood transfusion requirement in the immediate $24 \mathrm{~h}$ after intervention in the pelvic packing group. No significant differences in mortality or complication rate were observed between the two treatment protocols. Most current studies in the literature comparing angiography vs operating room (OR) do not necessarily address the question of comparative effectiveness - in some studies, the decision to go to the OR first was made on the likelihood of other sources of bleeding (i.e., +FAST), so despite the article title, they do not really compare equivalent situations [54]. The disadvantages of a management protocol reliant solely on angioembolization include delays due to the relative unavailability of the angiography suite/interventional radiologist and that it does not address venous pelvic bleeding. The contribution of venous hemorrhage to pelvic fracture exsanguination has been recognized in previous cadaveric and clinical studies [55-57].

Lastly, the use of tranexamic acid (TXA) to control hemorrhage in pelvic fractures remains controversial. Tranexamic acid use during elective arthroplasty surgery has been shown to reduce transfusion rates [58]. There are no studies to date regarding the use of tranexamic acid in pelvic fracture patients. The CRASH-2 trial examined the effects of TXA in trauma patients with or at risk of significant bleeding [59]. Their results showed a decreased risk of early all-cause mortality in patients who received TXA within $8 \mathrm{~h}$ of their injury. There was a statistically significant decrease in mortality due

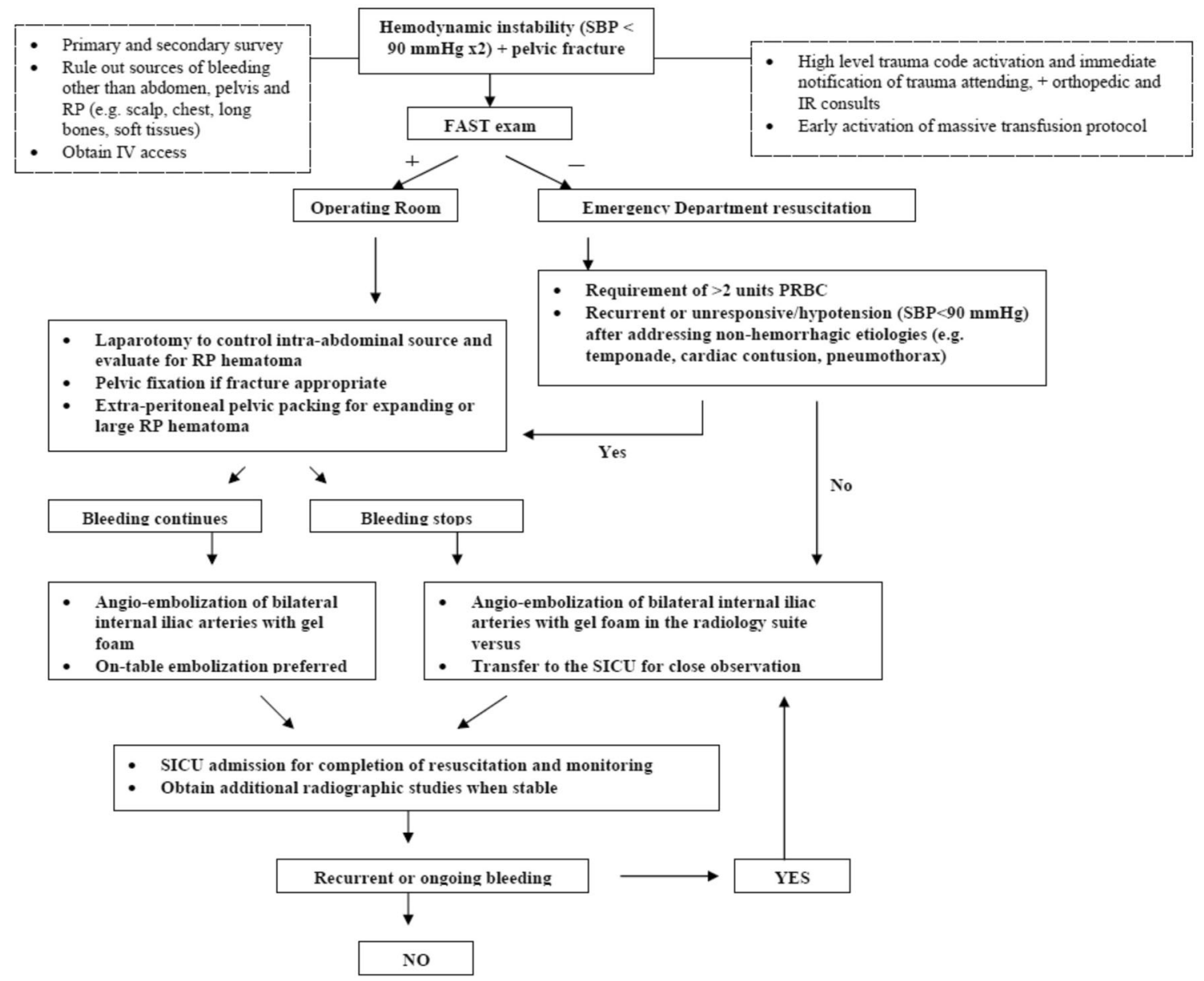

Fig. 9 Practical protocol to manage pelvic fractures in hemodynamically unstable patients utilizing retroperitoneal packing for hemorrhage control 
to bleeding; however, TXA did not appear to make any difference in transfusion requirements between the groups. Thus, a mechanistic explanation of the effect of TXA still remains to be elucidated and tempers its undisputed adoption into the treatment algorithm for bleeding pelvic fracture patients.

\section{Practical Protocol}

The protocol we utilize at our institutions is similar to that first described by Cothren et al. [24]. We prefer the use of retroperitoneal packing to control pelvic hemorrhage (Fig. 9). Patients with significant pelvic injury who fail to respond to 2 units of packed red blood cells are taken for emergent pelvic stabilization and retroperitoneal packing. Patients with a significant pelvic fracture but who respond to blood products are taken either to angiography or to the surgical intensive care unit for close monitoring, and their pelvic fracture is stabilized on a semi-urgent basis.

While we prefer the use of retroperitoneal packing, most centers in the USA favor a treatment strategy that focuses on angiography.

\section{Conclusion}

High-energy displaced fractures of the pelvis are a significant source of morbidity and mortality. The management of these complex injuries requires cooperation between the trauma and orthopedic surgeons. In patients with displaced pelvic ring injuries and hemodynamic instability, attention should be focused on aggressive resuscitation of the patient, early stabilization of the bony injury, and hemorrhage control through either angiography or retroperitoneal packing.

\section{Compliance with Ethics Guidelines}

Conflict of Interest Michael J. Weaver and Marilyn Heng declare that they have no conflict of interest.

Human and Animal Rights and Informed Consent This article does not contain any studies with human or animal subjects performed by any of the authors.

\section{References}

Papers of particular interest, published recently, have been highlighted as:

- Of importance

•• Of major importance

1. Sathy AK, Starr AJ, Smith WR, Elliott AE, Agudelo J, Reinert CM, et al. The effect of pelvic fracture on mortality after trauma: an analysis of 63,000 trauma patients. J Bone Joint Surg Am. 2009;91:2803-10.

2. Starr AJ, Griffin DR, Reinert CM, Frawley WH, Walker J, Whitlock SN, et al. Pelvic ring disruptions: prediction of associated injuries, transfusion requirement, pelvic arteriography, complications, and mortality. J Orthop Trauma. 2002;16(8):553-61.

3. Dente CJ, Feliciano DV, Rozycki GS, Wyrzykowski AD, Nicholas $\mathrm{JM}$, Salomone JP, et al. The outcome of open pelvic fractures in the modern era. Am J Surg. 2005;190(6):831-7.

4. Duchesne JC, Bharmal HM, Dini AA, Islam T, Schmieg RE, Simmons JD, et al. Open-book pelvic fractures with perineal open wounds: a significant morbid combination. Am Surg. 2009;75(12): 1227-33.

5. Inaba K, Sharkey PW, Stephen DJ, Redelmeier DA, Brenneman FD. The increasing incidence of severe pelvic injury in motor vehicle collisions. Injury. 2004;35(8):759-65.

6. Siegel JH, Dalal SA, Burgess AR, Young JW. Pattern of organ injuries in pelvic fracture: impact force implications for survival and death in motor vehicle injuries. Accid Anal Prev. 1990;22(5): 457-66.

7. Khanna P, Phan H, Hardy AH, Nolan T, Dong P. Multidisciplinary management of blunt pelvic trauma. Semin Interv Radiol. 2012;29(3):187-91.

8. Tosounidis TI, Giannoudis PV. Pelvic fractures presenting with haemodynamic instability: treatment options and outcomes. Surgeon. 2013;11(6):344-51.

9. ATLS Subcommittee, American College of Surgeons' Committee on Trauma, International ATLS working group. Advanced Trauma Life Support (ATLS): the ninth edition. J Trauma Acute Care Surg. 2013;74(5):1363-6.

10. Langford JR, Burgess AR, Liporace FA, Haidukewych GJ. Pelvic fractures: part 1 evaluation, classification, and resuscitation. J Am Acad Orthop Surg. 2013;21:448-57. This review article provides a detailed explanation of the evaluation and classification of pelvic fractures, specifically focusing on the Young-Burgess classification.

11. Hasankhani EG, Omidi-Kashani F. Treatment outcomes of open pelvic fractures associated with extensive perineal injuries. Clin Orthop Surg. 2013;5(4):263-8. This review article provides a detailed explanation of the evaluation and classification of pelvic fractures, specifically focusing on the Young-Burgess classification.

12. Cannada LK, Taylor RM, Reddix R, Mullis B, Moghadamian E, Erickson M. Southeastern Fracture Consortium. The Jones-Powell Classification of open pelvic fractures: a multicenter study evaluating mortality rates. J Trauma Acute Care Surg. 2013;74(3):901-6.

13. Dente CJ, Feliciano DV, Rozycki GS, Wyrzykowski AD, Nicholas JM, Salomone JP, et al. The outcome of open pelvic fractures in the modern era. Am J Surg. 2005;190(6):830-5.

14. Grotz MR, Allami MK, Harwood P, Pape HC, Krettek C, Giannoudis PV. Open pelvic fractures: epidemiology, current concepts of management and outcome. Injury. 2005;36(1):1-13.

15. Lunsjo K, Abu-Zidan FM. Does colostomy prevent infection in open blunt pelvic fractures? A systematic review. J Trauma. 2006;60(5):1145-8.

16. Pell M, Flynn WJ, Seibel RW. Is colostomy always necessary in the treatment of open pelvic fractures? J Trauma. 1998;45(2):371-3.

17. Gomez RG, Mundy T, Dubey D, El-Kassaby AW, Firdaoessaleh, Kodama R, Santucci R. SIU/ICUD Consultation on Urethral Strictures: Pelvic Fracture Urethral Injuries. Urology. 2014; 83(3 Suppl): S48-S58

18. Hsieh CH, Chen RJ, Fang JF, Lin BC, Hsu YP, Kao JL, et al. Diagnosis and management of bladder injury by trauma surgeons. Am J Surg. 2002;184:143-7.

19. Berber O, Emeagi C, Perry M, Rickman MS. Failure of conventional retrograde cystography to detect bladder ruptures in pelvic trauma. J Orthop Traumatol. 2011;12(1):57-60. 
20. Guillamondegui OD, Pryor JP, Gracias VH, Gupta R, Reilly PM, Schwab CW. Pelvic radiography in blunt trauma resuscitation: a diminishing role. J Trauma. 2002;53:1043-7.

21. Kessel B, Sevi R, Jeroukhimov I, Kalganov A, Khashan T, Ashkenazi I, et al. Is routine portable pelvic X-ray in stable multiple trauma patients always justified in a high technology era? Inj Int $\mathrm{J}$ Care Injured. 2007;38:559-63.

22. Fu C-Y, Wang S-Y, Hsu Y-P, Liao C-H, Lin B-C, Kang S-C, et al. The diminishing role of pelvic $\mathrm{X}$-rays in the management of patients with major torso injuries. Am J Emerg Med. 2014;32(1):18-23.

23. Dalal SA, Burgess AR, Siegel JH, Young JW, Brumback RJ, Poka A, et al. Pelvic fracture in multiple trauma: classification by mechanism is key to pattern of organ injury, resuscitative requirements, and outcome. J Trauma. 1989;29(7):981-1002.

24. Cothren CC, Osborn PM, Moore EE, Morgan SJ, Johnson JL, Smith WR. Preperitoneal pelvic packing for hemodynamically unstable pelvic fractures: a paradigm shift. J Trauma. 2007;62(4):8349.

25.• Cheng M, Cheung M-T, Lee K-Y, Lee K-B, Chang SC, Wu AC, et al. Improvement in institutional protocols leads to decreased mortality in patients with haemodynamically unstable pelvic fractures. Emerg Med J. 2013. doi:10.1136/emermed-2012-2022009. This was a retrospective cohort study comparing mortality in patients with unstable pelvic fractures treated either with preangiography, with angiography, or with pelvic packing. Early mortality within 30 days was significantly decreased in the pelvic packing group; multivariate logistic regression identified pelvic packing as an independent predictive factor of survival at $24 \mathrm{~h}$. Early mortality within 30 days was significantly decreased in the pelvic packing group; multivariate logistic regression identified pelvic packing as an independent predictive factor of survival at $24 \mathrm{~h}$.

26. Ip KC, Lee KB. Standardised multidisciplinary protocol for haemodynamically unstable pelvic fractures. J Orthop Surg (Hong Kong). 2014;22(2):177-80.

27. Perkins ZB, Maytham GD, Koers L, Bates P, Brohi K, Tai NRM. Impact on outcome of a targeted performance improvement programme in haemodynamically unstable patients with a pelvic fracture. Bone Joint J. 2014;96-B:1090-7.

28. Grimm MR, Vrahas MS, Thomas KA. Pressure-volume characteristics of the intact and disrupted pelvic retroperitoneum. J Trauma. 1998;44(3):454-9.

29.• Morrison CA, Carrick MM, Norman MA, Scott BG, Welsh FJ, Tsai $\mathrm{P}$, et al. Hypotensive resuscitation strategy reduces transfusion requirements and severe postoperative coagulopathy in trauma patients with hemorrhagic shock: preliminary results of a randomized controlled trial. J Trauma. 2011;70:652-63. Trauma patients in shock requiring laparotomy or thoratomy were randomized to two groups regarding intraoperative resuscitation: 1) low mean arterial pressure target (MAP) of $50 \mathrm{mmHg} v \mathrm{~s}$ 2) high MAP target of $65 \mathrm{mmHg}$. Patients in the LMAP received less blood products and IV fluids intraoperatively, were less likely to develop perioperative coagulopathy, and showed lower early $(<24$ h) perioperative mortality. Mortality at 30 days was not significantly different between the groups.

30. Leenen M, Scholz A, Lefering R, Flohe S, On behalf of the Committee on Emergency Medicine, Intensive Care and Trauma Management of the German Trauma Society. Limited volume resuscitation in hypotensive elderly multiple trauma is safe and prevents early clinical dilutive coagulopathy — a matched pair analysis from TraumaRegister DGU. Inj Int J Care Injured. 2014;45S:S5963.

31. Borgman M, Spinella PC, Perkins JG, Grathwohl KW, Repine T, Beekley AC, et al. The ratio of blood products transfused affects mortality in patients receiving massive transfusions at a combat support hospital. J Trauma. 2007;63:805-13.
32. Holcomb JB, Wade CE, Michalek JE, Chisholm GB, Zarzabal LA, Schreiber MA, et al. Increased plasma and platelet to red blood cell ratios improves outcome in 466 massively transfused civilian trauma patients. Ann Surg. 2008;248:447-58.

33. Holcomb JB, del Junco DJ, Fox EE, Wade CE, Cohen MJ, Schreiber MA, et al. The Prospective, Observational, Multicenter, Major Trauma Transfusion (PROMMTT) Study. JAMA Surg. 2013;148(2):127-36.

34. Manson T, O'Toole RV, Whitney A, Duggan B, Sciadini M, Nascone J. Young-Burgess classification of pelvic ring fractures: does it predict mortality, transfusion requirements, and nonorthopaedic injuries? J Orthop Trauma. 2010;24:603-9.

35. Osterhoff G, Scheyerer MJ, Fritz Y, Bouaicha S, Wanner GA, Simmen HP, et al. Comparing the predictive value of the pelvic ring injury classification systems by Tile and by Young and Burgess. Inj Int J Care Injured. 2014;45:742-7.

36. Dyer GSM, Vrahas MS. Pathophysiology and acute management of haemorrhage in pelvic fracture. Inj Int J Care Injured. 2006;37:602_13 .

37. White CE, Hsu JR, Holcomb JB. Haemodynamically unstable pelvic fractures. 2009; 40 (10): 1023-1030.

38. Rajab TK, Weaver MJ, Havens JM. Technique for temporary pelvic stabilization after trauma. N Engl J Med. 2013;369:e22(1)-4). This is an article with an accompanying video demonstrating the proper technique for sheeting an unstable pelvis for temporization of hemorrhage from pelvic fracture. Key points include the centering of the sheet at the level of the greater trochanters, securing of the tightened sheet with Kelly clamps, and postsheeting monitoring to avoid the development of pressure ulceration.

39. Spanjersberg WR, Knops SP, Schep NW, van Lieshout EM, Patka $\mathrm{P}$, Schipper IB. Effectiveness and complications of pelvic circumferential compression devices in patients with unstable pelvic fractures: a systematic review of literature. Injury. 2009;40:1031-5.

40. Rommens PM: External fixation for the injured pelvic ring. In Tile M, Helfet D, Kellam J (eds). Fractures of the pelvis and acetabulum. Ed 3. Philadelphia, Lippincott, Williams \& Wilkins. 2003. 203216 ,

41. Giannoudis PV, Pape HC. Damage control orthopaedics in unstable pelvic ring injuries. Injury. 2004;35:671-7.

42. Mason WT, Khan SN, James CL, Chesser TJ, Ward AJ. Complications of temporary and definitive external fixation of pelvic ring injuries. Injury. 2012;36(5):599-604.

43. Sellei RM, Schandelmaier P, Kobbe P, Knobe M, Pape HC. Can a modified anterior external fixator provide posterior compression of AP compression type III pelvic injuries? Clin Orthop Relat Res. 2013;471:2862-8.

44. Gansslen A, Giannoudis P, Pape HC. Hemorrhage in pelvic fracture: who needs angiography? Curr Opin Crit Care. 2003;9(6):51523.

45. Heini PF, Witt J, Ganz R. The pelvic C-clamp for the emergency treatment of unstable pelvic ring injuries. Injury. 1996;27 Suppl: SA38-45.

46. Koller H, Balogh ZJ. Single training session for first time pelvic Cclamp users: correct pin placement and frame assembly. Injury. 2012;43(4):436-9.

47. Enninghorst N, Toth L, King KL, McDougall D, Mackenzie S, Balogh ZJ. Acute definitive internal fixation of pelvic ring fractures in polytrauma patients: a feasible option. J Trauma. 2010;68:93541.

48. Hawkins L, Pomerantz M, Eiseman B. Laparotomy at the time of pelvic fracture. J Trauma. 1970;10:610-23.

49. Flint LM, Brown A, Richardson JD, Polk HC. Definitive control of bleeding from severe pelvic fractures. Ann Surg. 1979;189:709-16.

50. Burlew CC, Moore EE, Smith WR, Johnson JL, Biffl WL, Barnett $\mathrm{CC}$, et al. Preperitoneal pelvic packing/external fixation with secondary angioembolization: optimal care for life-threathening 
hemorrhage from unstable pelvic fracture. J Am Coll Surg. 2011;212:628-37.

51. Salim A, Teixeria PG, DuBose J, Ottochian M, Inaba K, Margulies DR, et al. Predictors of positive angiography in pelvic fractures: a prospective study. J Am Coll Surg. 2008;207(5):656-62.

52. Papkostidis C, Kanakaris N, Dimitriou R, Giannoudis. The role of arterial embolization in controlling pelvic fracture haemorrhage: a systematic review of the literature. Euro J Radiol. 2012;82:897904.

53.• Osborn PM, Smith WR, Moore EE, Cothren CC, Morgan SJ, Williams AE, et al. Direct retroperitoneal pelvic packing vs. angiography: a comparison of two management protocols for haemodynamically unstable pelvic fractures. Inj Int J Care Injured. 2009;40:54-60. A retrospective review of hemodynamically unstable pelvic fracture patients treated with either pelvic packing or early pelvic angiography was performed. The median time to treatment (either packing or angiography, respectively) was shorter in the packing group (45 min vs 130 min for the angiography group). The packing group also had a lower rate of blood transfusions in the $24 \mathrm{~h}$ postoperative period. There was no significant difference in the in-hospital mortality between the groups.
54. Thorson CM, Ryan ML, Otero CA, Vu T, Borja MJ, Jose J, et al. Operating room or angiography suite for hemodynamically unstable pelvic fractures? J Trauma. 2012;72:364-72.

55. Ertel W, Keel M, Eid K, Platz A, Trentz O. Control of severe hemorrhage using C-clamp and pelvic packing in multiply injured patients with pelvic ring disruption. J Orthop Trauma. 2001;15(7): 468-74.

56. Baque P, Trojani C, Delotte J, Sejor E, Senni-Buratti M, de Baque F, et al. Anatomical consequences of "open-book" pelvic ring disruption: a cadaver experimental study. Surg Radiol Anat. 2005;27(6): 487-90.

57. Kataoka Y, Maekawa K, Nishimaki H, Soma SK. Iliac vein injuries in hemodynamically unstable patients with pelvic fracture caused by blunt trauma. J Trauma. 2005;58(4):704-10.

58. Wind TC, Barfield WR, Moskal JT. The effective of tranexamic acid on transfusion rate in primary total hip arthroplasty. J Arthroplasty. 2014;29(2):387-9.

59. CRASH-2 Trial Collaborators. Effects of tranexamic acid on death, vascular occlusive events, and blood transfusion in trauma patients with significant haemorrhage (CRASH-2): a randomized, placebocontrolled trial. Lancet. 2010;376:23-32. 